\title{
RELAÇÃO ENTRE APOIO SOCIAL E BEM-ESTAR SUBJETIVO EM IDOSOS: REVISÃO SISTEMÁTICA
}

\section{Relationship between social support and subjective well-being in older people: systematic review Relación entre apoyo social y bienestar subjetivo de mayores: revisión sistemática}

\author{
Luiz Fellipe Dias da Rocha \\ Universidade do Estado do Rio de Janeiro - UERJ - Rio de Janeiro (RJ) - Brasil
}

\section{Evlyn Rodrigues Oliveira}

Universidade do Estado do Rio de Janeiro - UERJ - Rio de Janeiro (RJ) - Brasil

\author{
Marcia Maria Peruzzi Elia da Mota \\ Universidade do Estado do Rio de Janeiro - UERJ - Rio de Janeiro (RJ) - Brasil
}

\section{RESUMO}

Objetivo: Investigar as evidências científicas acerca da associação entre apoio social e bem-estar subjetivo em idosos. Métodos: Revisão de literatura advinda da busca de artigos publicados nos últimos dez anos, na base de dados do Portal de Periódicos da Capes. Os descritores utilizados foram: social support AND well being OR life satisfaction OR positive affect OR negative affect. Incluíram-se no estudo artigos completos publicados em periódicos, nos idiomas inglês, português e espanhol, que tinham como objetivo avaliar a predição do apoio social no bem-estar subjetivo. Resultados: A seleção inicial resultou em 311 artigos. Após análise e submissão aos critérios de inclusão e exclusão, restaram dez artigos, sendo todos em língua inglesa. Todos os estudos selecionados encontraram associações entre apoio social e bem-estar subjetivo. Em suma, quatro tipos de impactos foram observados: (a) direto e positivo; (b) direto e negativo; (c) direto e mediador; e (d) indireto e mediado. Conclusão: Os resultados dessa revisão apontaram que a relação entre apoio social e bem-estar subjetivo é, de modo geral, positiva. Portanto, a tendência dos artigos avaliados foi a de que o apoio eleva o bem-estar dos idosos, levando-os a avaliar suas vidas de modo mais positivo, a experimentar mais afetos agradáveis e menos afetos desprazerosos.

Descritores: Idoso; Apoio Social; Qualidade de Vida.

\begin{abstract}
Objective: To investigate the scientific evidence on the association between social support and subjective well-being in older people. Methods: Systematic literature review of articles published in the last ten years available in the database of the Capes Journals Portal. The descriptors used were: social support AND well being OR life satisfaction OR positive affect OR negative affect. The study included full articles published in English, Portuguese and Spanish aimed at assessing the prediction of social support in subjective well-being. Results: Initial selection resulted in 311 articles. After analysis and application of inclusion and exclusion criteria, ten articles remained; all the articles were in English. All the selected studies found associations between social support and subjective well-being. In summary, four types of impacts were observed: (a) direct and positive; (b) direct and negative; (c) direct and mediator; and (d) indirect and mediated. Conclusion: The results of this review pointed out that the relationship between social support and subjective well-being is generally positive. Therefore, the articles showed that the support improves older people's well-being, leading them to rate their lives more positively and to experience more pleasant affects and less unpleasant affects.
\end{abstract}

Descriptors: Aged; Social Support; Quality of Life. 


\section{RESUMEN}

Objetivo: Investigar las evidencias cientificas sobre la asociación entre el apoyo social y el bienestar subjetivo de mayores. Métodos: Revisión de la literatura con la búsqueda de artículos publicados en los últimos diez años en la base de datos del Portal de Periódicos de Capes. Los descriptores utilizados fueron: social support AND well being OR life satisfaction OR positive affect OR negative affect. Se incluyeron en el estudio los artículos completos publicados en periódicos en los idiomas inglés, portugués y español que tenían el objetivo de evaluar la predicción del apoyo social en el bienestar subjetivo. Resultados: La selección inicial fue de 311 artículos. Tras el análisis y la observación de los criterios de inclusión y exclusión se quedaron diez artículos todos en el idioma inglés. En todos los estudios elegidos se encontraron asociaciones entre el apoyo social y el bienestar subjetivo. En resumen, fueron observados cuatro tipos de impactos: (a) directo y positivo; (b) directo y negativo; (c) directo y mediador; y (d) indirecto y mediado. Conclusión: Los resultados de esa revisión apuntan a que la relación entre el apoyo social y el bienestar subjetivo es, en general, positiva. Por lo tanto, la tendencia de los artículos evaluados fue que el apoyo mejora el bienestar de los mayores llevándolos a evaluar sus vidas de manera más positiva y experimentar más a los afectos agradables y menos a los afectos que no causan placer.

Descriptores: Anciano; Apoyo Social; Calidad de Vida.

\section{INTRODUÇÃO}

O Bem-Estar Subjetivo (BES) é encarado como a maneira como pessoa avalia sua própria vida e seus diferentes domínios em termos cognitivos e afetivos. Isto é, o BES é um construto de estrutura tripartite composto por um componente cognitivo (satisfação com a vida - SV) e dois afetivos (afetos positivos - AP; e negativos - AN) ${ }^{(1-3)}$. Para se eleger um nível de Bem-Estar Subjetivo elevado, consideram-se experiências emocionais positivas frequentes, rara experiência emocional negativa, e alto nível de satisfação com a vida ${ }^{(4)}$.

Diversos autores têm apontado o bem-estar subjetivo como um indicador essencial para avaliar o envelhecimento bemsucedido. Isso porque a qualidade de vida vai muito além dos indicadores objetivos, como status econômico ou critérios sociodemográficos. Ela engloba indicadores sociais que incluem critérios de julgamento pessoal do bem-estar, modo pelo qual se percebe os diversos domínios de sua vida ${ }^{(5-7)}$.

O bem-estar subjetivo é uma importante variável a ser considerada em estudos gerontológicos, pois informa o grau de adaptação dos idosos diante das circunstâncias objetivas da vida. Deste modo, é imprescindível uma melhor compreensão do comportamento desse construto psicológico na terceira idade para que medidas sejam implementadas no sentido de aumentar o bem-estar dessa população e levá-los a viver uma vida que vale a pena ser vivida ${ }^{(8)}$.

Uma das formas de elevar o BES de uma pessoa é conhecendo as variáveis que estão associadas a ele para, então, intervir sobre elas. Tanto fatores internos ao indivíduo (modelo top-down) como fatores externos (modelo bottom-up) têm se mostrado importantes preditores do bem-estar ${ }^{(2)}$. No primeiro caso, referente ao conjunto de fatores intrínsecos, a literatura tem evidenciado a substancial influência da personalidade, da espiritualidade, do lócus de controle, das estratégias de coping, das crenças autorreferenciadas, da interpretação subjetiva dos eventos, entre outros ${ }^{(9-12)}$.

No tocante ao conjunto de fatores extrínsecos, as variáveis mais pesquisadas são a saúde, a renda econômica, o nível educacional, a condição conjugal, os eventos de vida, entre outras ${ }^{(10,13,14)}$. Além das variáveis citadas anteriormente, o apoio social ganha certo destaque no modelo bottom-up por apresentar uma forte associação com o BES ao longo do ciclo vital, mas especialmente na longevidade, em que os idosos tendem a sofrer perdas de papeis sociais e demonstram maior propensão à vulnerabilidade e à fragilidade ${ }^{(6,10)}$.

Não existe um consenso na literatura quanto à definição do apoio social (AS), contudo ele é estudado, na maioria das vezes, a partir do seu aspecto funcional. Portanto, o apoio social é encarado como qualquer tipo de comportamento que tenha por objetivo suprir as necessidades de outras pessoas e/ou grupos e que resulte em efeitos positivos sobre a vida de quem o recebe ${ }^{(15)}$.

Embora a provisão de apoio seja um comportamento essencial para o suprimento de necessidades básicas, sociais e psicológicas, prover apoio não é um processo simples e depende de uma série de competências cognitivas e emocionais como: (a) reconhecer que uma pessoa precisa de ajuda; (b) avaliar se essa pessoa está aberta para receber ajuda; (c) analisar que tipo de ajuda ela está precisando; (d) decidir ajudar; e, (e) decidir como ajudar.

Por se tratar de um construto complexo, muitas são as formas de estudá-lo. Inúmeros pesquisadores optam por utilizar diferentes perspectivas de apoio social. Um autor ${ }^{(6)}$ aponta a existência de, pelo menos, três delas: perceived support, enacted support (ou received support), provided support. Essas dimensões costumam aparecer na literatura brasileira e em outros países de língua portuguesa como respectivamente: Apoio Percebido, Apoio Recebido e Apoio Provido.

O apoio recebido, como o próprio termo apresenta, refere-se ao apoio que a pessoa relata de fato estar recebendo atualmente ou ter recebido em algum momento de sua vida. Já o apoio percebido é definido como o apoio que uma pessoa acredita estar disponível caso ela precise. O apoio provido avalia, a partir da perspectiva do provedor, a quantidade e/ou a qualidade do apoio que uma pessoa provê ou acredita estar disposta a prover a outras pessoas. 
Existe também a possibilidade de trabalhar o apoio social em dimensões que fazem referencia ao conteúdo da ajuda, tais como o apoio instrumental/ material, que diz respeito ao fornecimento de recursos materiais ou assistência prática em atividades concretas; o apoio emocional, relacionado a comportamentos que gerem no outro a sensação de ser valorizado; e o apoio informacional, fornecimento de informações ou conselhos úteis ${ }^{(15)}$.

Discriminar diferentes dimensões de apoio pode ser importante na medida em que cada uma delas pode estar relacionada de forma distinta com outros construtos indicadores de saúde. Essa questão da multidimensionalidade do AS se apresenta de modo controverso nas pesquisas empíricas e tal fato pode ser explicado pelo fato das dimensões estarem intimamente correlacionadas. "As pessoas que recebem um determinado tipo de apoio também têm maior probabilidade de receberem outros tipos de ajuda", o que provoca uma maior dependência entre as dimensões ${ }^{(16)}$.

Em um estudo prévio ${ }^{(17)}$ aponta-se para as diferentes formas como as pessoas entendem o significado dos itens das escalas que se destinam a mensurar o AS como uma das possíveis causas na dificuldade em discriminar suas dimensões. De acordo com os envolvidos na relação de ajuda, o apoio social pode ser definido ainda como formal ou informal. O apoio formal está relacionado às instituições (governamentais ou não) e interações profissionais (por exemplo, com médicos, enfermeiros, cuidadores e assistentes sociais). Já o apoio informal envolve pessoas que fazem parte da rede social do sujeito em questão, como membros da família, amigos e vizinhos ${ }^{(18)}$.

Tendo em vista que tanto o apoio social como o bem-estar subjetivo são construtos complexos, o presente estudo tem como objetivo investigar as evidências científicas acerca da associação entre apoio social e o bem-estar subjetivo em idosos.

\section{MÉTODOS}

Para a presente revisão sistemática, foi utilizada a base de dados do Portal de Periódicos da CAPES, que agrega diferentes indexadores relevantes de revistas científicas nacionais e internacionais, tais como PubMed, SciELO e Pepsic. A busca ocorreu no mês de janeiro de 2017. Nela, foram selecionados artigos científicos publicados nos últimos 10 anos que apresentassem em seus títulos as palavras-chave "social support" AND "well being" OR "life satifaction" OR "positive affect" OR "negative affect". A seleção inicial resultou em 311 artigos, conforme indicado na Figura 1. Após a aplicação dos critérios de exclusão ("não pertencer aos idiomas inglês, espanhol ou português" e "não possuir resumo disponível no Portal Capes"), 129 artigos foram retirados da amostra. Com a remoção dos artigos que apareciam em duplicata, 167 seguiram para análise dos resumos.

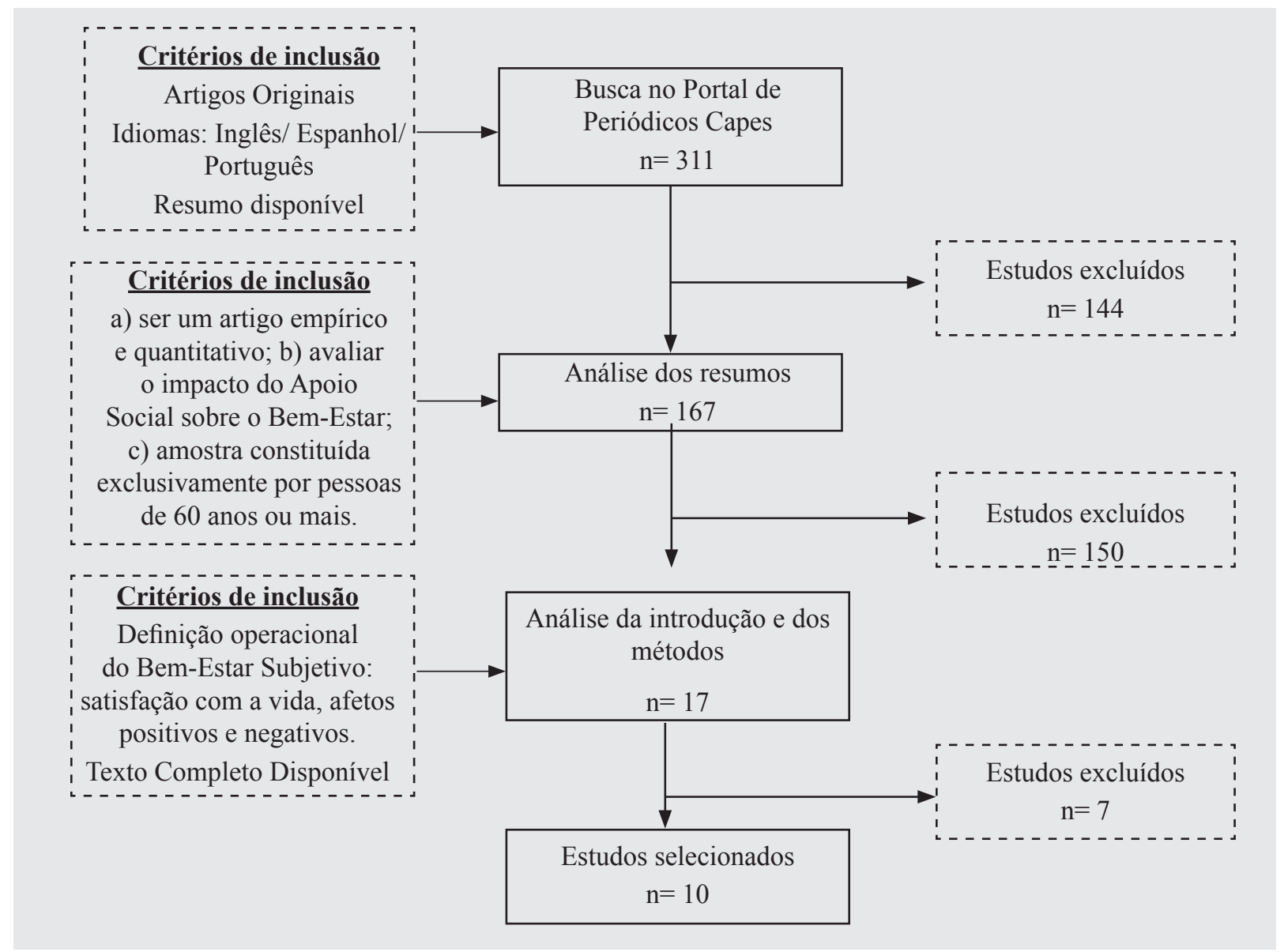

Figura 1 - Fluxograma da busca bibliográfica. 
Nesta etapa, dois juízes avaliaram os resumos separadamente, a fim de verificar se atendiam aos seguintes critérios de inclusão: a) ser um artigo empírico e quantitativo; b) avaliar o impacto do apoio social sobre o bem-estar; c) amostra constituída exclusivamente por pessoas de 60 anos ou mais. Não havendo discordâncias entre as avaliações, 17 artigos foram selecionados e seguiram para análise das introduções e metodologias com o objetivo de verificar se o estudo examinou especificamente a influência do apoio social sobre as facetas do bem-estar subjetivo, a saber, satisfação com a vida, afetos positivos e negativos. Como na análise dos resumos, essa avaliação ocorreu com a avaliação de dois juízes de forma independente. Na comparação dos resultados, em caso de divergência, buscou-se o consenso. Dois artigos não estavam disponíveis integralmente e saíram da amostra. Após avaliação, removeram-se mais cinco artigos. Ao cabo de todas as etapas, dez artigos compuseram o banco final.

Realizou-se, então, a leitura analítica e integral de cada estudo visando identificar as ideias-chave, investigar estratégias metodológicas e sintetizar os resultados encontrados. Para facilitar a análise, classificação e categorização dos artigos, os dados foram dispostos em um formulário construído pelos autores com base no instrumento Guidelines for Critical Review Form: Quantitative Studies ${ }^{(19)}$. Todas as pesquisas apresentaram boa qualidade metodológica.

\section{RESULTADOS}

Todos os estudos foram realizados em países orientais, sendo sete na China (70\%), dois no Nepal (20\%) e um na Malásia (10\%). A maioria deles (60\%) foi publicada nos últimos cinco anos. Todas as pesquisas apresentaram desenho transversal nãoexperimental. O tamanho da amostra variou entre 133 e 1880 idosos, com variação de idade média entre 63 e 79 anos.

Os estudos selecionados foram organizados nos seguintes eixos: autores, ano de publicação, local de pesquisa, tipo de estudo, tamanho da amostra, média de idade dos participantes (Quadro I), objetivos do estudo, escalas utilizadas para mensurar o apoio social e respectivas confiabilidades, instrumentos utilizados para avaliação do bem-estar subjetivo e respectivas confiabilidades (Quadro II), metodologia de análise dos dados e principais resultados (Quadro III).

Quadro I - Características dos estudos selecionados sobre apoio social e bem-estar subjetivo entre 2007 e 2017.

\begin{tabular}{|c|c|c|c|c|c|}
\hline Autores & $\begin{array}{c}\text { Ano de } \\
\text { publicação }\end{array}$ & Local de pesquisa & $\begin{array}{l}\text { Tipo de } \\
\text { estudo }\end{array}$ & Amostra & $\begin{array}{c}\text { Média de idade dos } \\
\text { participantes (Desvio Padrão) }\end{array}$ \\
\hline Kooshiar et al. ${ }^{(20)}$ & 2012 & $\begin{array}{l}\text { Região peninsular, } \\
\text { Malásia }\end{array}$ & Transversal & 1880 & $69,79(\mathrm{DP}=7,36)$ \\
\hline Wang $^{(23)}$ & 2014 & Hefei, China & Transversal & 314 & $65,32(\mathrm{DP}=3,16)$ \\
\hline Tu; Yang ${ }^{(24)}$ & 2016 & Dez cidades da China & Transversal & 335 & NI \\
\hline $\operatorname{Tian}^{(25)}$ & 2016 & NI, China & Transversal & 429 & $63,22(\mathrm{DP}=3,23)$ \\
\hline $\mathrm{Lou}^{(28)}$ & 2010 & Hong Kong, China & Transversal & 215 & $79,3(\mathrm{DP}=6,9)$ \\
\hline Li et al. ${ }^{(30)}$ & 2014 & Beijing, China & Transversal & 700 & NI \\
\hline Peng et al. ${ }^{(31)}$ & 2015 & Shenzhen, China & Transversal & 133 & $65,46(\mathrm{DP}=4,23)$ \\
\hline Phillips et al. ${ }^{(33)}$ & 2008 & Hong Kong, China & Transversal & 518 & NI \\
\hline Chalise et al. ${ }^{(34)}$ & 2007 & Katmandu, Nepal & Transversal & 509 & NI \\
\hline Chalise $^{(35)}$ & 2010 & Katmandu, Nepal & Transversal & 332 & $68,95(\mathrm{DP}=7,45)$ \\
\hline
\end{tabular}

Nota: DP: Desvio padrão; NI: Não informado

Quadro II - Características dos estudos selecionados sobre apoio social e bem-estar subjetivo entre 2007 e 2017.

\begin{tabular}{|c|c|c|}
\hline Autores & $\begin{array}{c}\begin{array}{c}\text { Escalas de apoio social } \\
\text { (confiabilidade) }\end{array} \\
\end{array}$ & $\begin{array}{c}\text { Escalas de bem-estar } \\
\text { subjetivo (confiabilidade) }\end{array}$ \\
\hline Kooshiar et al. ${ }^{(20)}$ & $\begin{array}{l}\text { Examinar as relações entre tipos Medical Outcomes Study Social } \\
\text { de arranjos domiciliares, apoio Support Survey scales }(\mathrm{a}=0,95) \\
\text { social e satisfação com a vida }\end{array}$ & $\begin{array}{l}\text { Philadelphia Geriatric Center } \\
\text { Morale Scale }(\mathrm{a}=0,76)\end{array}$ \\
\hline Wang $^{(23)}$ & $\begin{array}{l}\text { Examinar as relações entre rede Perceived Social Support Scale } \\
\text { social, apoio social percebido e }(\mathrm{a}=0,88) \\
\text { bem-estar subjetivo }\end{array}$ & $\begin{array}{l}\text { Subjective Well Being scale } \\
\text { (alpha de subdomínios entre } \\
0,79 \text { e } 0,84 \text { ) }\end{array}$ \\
\hline Tu; Yang ${ }^{(24)}$ & $\begin{array}{l}\text { Explorou as relações entre o Chinese Social Support Rating } \\
\text { autocontrole, o apoio social e o Scale }(\mathrm{a}=0,72) \\
\text { bem-estar subjetivo }\end{array}$ & $\begin{array}{l}\text { Subjective Well Being scale } \\
\text { (alpha de subdomínios entre } \\
0,70 \text { e } 0,74 \text { ) }\end{array}$ \\
\hline $\operatorname{Tian}^{(25)}$ & $\begin{array}{l}\text { Avaliar o efeito do apoio social Intergenerational Social Support } \\
\text { intergeracional sobre o bem-estar } \begin{array}{l}\text { Scale para apoio provido e } \\
\text { subjetivo }\end{array} \text { recebido }(0,774)\end{array}$ & $\begin{array}{l}\text { Subjective Well Being scale } \\
\text { (alpha de subdomínios entre } \\
0,76 \text { e } 0,84 \text { ) }\end{array}$ \\
\hline
\end{tabular}




\begin{tabular}{|c|c|c|c|}
\hline $\mathrm{Lou}^{(28)}$ & $\begin{array}{l}\text { Investigar a associação entre } \\
\text { apoio social em relação aos netos } \\
\text { e satisfação com a vida }\end{array}$ & $\begin{array}{l}\text { Escalas próprias para apoio }(\mathrm{NI}) \text { e } \\
\text { qualidade do relacionamento }(\mathrm{a}= \\
0,76)\end{array}$ & $\begin{array}{l}\text { Life Satisfaction Scale for } \\
\text { Chinese Old Adult }(\mathrm{a}=0,90)\end{array}$ \\
\hline Li et al. ${ }^{(30)}$ & $\begin{array}{l}\text { Avaliar o impacto do apoio social } \\
\text { sobre a experiência de afetos } \\
\text { positivos e negativos }\end{array}$ & $\begin{array}{l}\text { Escala própria para apoio social } \\
\text { provido e recebido } \\
(\mathrm{a}=0,84)\end{array}$ & $\begin{array}{l}\text { The Positive and Negative } \\
\text { Affect Scales }(\mathrm{a}=0,75 \text { e } 0,87 \text {, } \\
\text { respectivamente) }\end{array}$ \\
\hline Peng et al. ${ }^{(31)}$ & $\begin{array}{l}\text { Avaliar o papel dos apoios } \\
\text { intergeracional e externo sobre } \\
\text { a dimensão afetiva do bem-estar } \\
\text { subjetivo }\end{array}$ & $\begin{array}{l}\text { Escalas próprias para apoio } \\
\text { intergeracional provido }(\mathrm{a}=0,91) \\
\mathrm{e} \text { recebido }(\mathrm{a}=0,93) / \text { Perceived } \\
\text { Social Support Scale adaptada } \\
\text { para apoio externo }(\mathrm{a}=0,91)\end{array}$ & $\begin{array}{l}\text { Memorial University } \\
\text { Newfoundland Scale } \\
\text { Happiness }(\mathrm{a}=0,84)\end{array}$ \\
\hline Phillips et al. ${ }^{(33)}$ & $\begin{array}{l}\text { Analisar o impacto da satisfação } \\
\text { com o apoio recebido sobre a } \\
\text { dimensão afetiva do BES }\end{array}$ & $\begin{array}{l}\text { Escala própria de item único para } \\
\text { satisfação com apoio recebido } \\
(\mathrm{a}=\mathrm{NI})\end{array}$ & $\begin{array}{l}\text { Itens extraídos do instrumento } \\
\text { World Health Organization } \\
\text { Quality of Life }(\mathrm{a}=\mathrm{NI})\end{array}$ \\
\hline Chalise et al. ${ }^{(34)}$ & $\begin{array}{l}\text { Analisar a influencia do apoio } \\
\text { social sobre o bem-estar subjetivo } \\
\text { e a solidão/ isolamento }\end{array}$ & $\begin{array}{l}\text { Escalas próprias para apoio } \\
\text { provido e apoio recebido (alpha } \\
\text { entre } 0,71 \text { e } 0,93 \text { ) }\end{array}$ & $\begin{array}{l}\text { Life Satisfaction Index } \mathrm{K}(\mathrm{a}= \\
0,80 \text { para subdomínio cognitivo } \\
\mathrm{e} a=0,92 \text { para subdomínio } \\
\text { afetivo) }\end{array}$ \\
\hline Chalise $^{(35)}$ & $\begin{array}{l}\text { Analisar a influencia do apoio } \\
\text { social sobre o bem-estar subjetivo } \\
\text { e a solidão/ isolamento }\end{array}$ & $\begin{array}{l}\text { Escalas próprias para apoio } \\
\text { provido }(\mathrm{a}=0,68) \text { e apoio recebido } \\
(\mathrm{a}=0,94)\end{array}$ & $\begin{array}{l}\text { Life Satisfaction Index } \mathrm{K}(\mathrm{a}= \\
0,80 \text { para subdomínio cognitivo } \\
\mathrm{e} a=0,92 \text { para subdomínio } \\
\text { afetivo) }\end{array}$ \\
\hline
\end{tabular}

Nota: a: coeficiente alpha de Cronbach; NI: Não informado

Quadro III - Características dos estudos selecionados sobre apoio social e bem-estar subjetivo entre 2007 e 2017.

\begin{tabular}{|c|c|c|}
\hline Autores & $\begin{array}{l}\text { Análises } \\
\text { dos dados }\end{array}$ & Resultados principais \\
\hline \multirow[b]{2}{*}{$\begin{array}{l}\text { Kooshiar et } \\
\text { al. }{ }^{(20)}\end{array}$} & $\begin{array}{l}\text { Correlações } \\
\text { de Pearson }\end{array}$ & O apoio social apresentou correlação significativa com satisfação com a vida $(r=0,36, p<0,001)$. \\
\hline & $\begin{array}{l}\text { Modelagem } \\
\text { de equação } \\
\text { estrutural }\end{array}$ & Houve uma relação significativa entre apoio social e satisfação com a vida $(\beta=0,28, p<0,01)$. \\
\hline \multirow[b]{2}{*}{ Wang $^{(23)}$} & $\begin{array}{l}\text { Correlações } \\
\text { de Pearson }\end{array}$ & NI \\
\hline & $\begin{array}{l}\text { Modelagem } \\
\text { de equação } \\
\text { estrutural }\end{array}$ & $\begin{array}{l}\text { O apoio social percebido funcionou como uma variável mediadora entre a rede social e o bem- } \\
\text { estar subjetivo }(\chi 2(12, \mathrm{n}=314)=31,49, \chi 2 / \mathrm{df}=2,62 ; \mathrm{RMSEA}=0,072 ; \text { SRMR }-=0,063 ; \text { and } \\
\text { CFI }=0,98)\end{array}$ \\
\hline \multirow[b]{2}{*}{ Tu; Yang ${ }^{(24)}$} & $\begin{array}{l}\text { Correlações } \\
\text { de Pearson }\end{array}$ & $\begin{array}{l}\text { O apoio social se mostrou significativamente corelacionado com satisfação com a vida ( } r= \\
0,16 ; p<0,01) \text {, afetos positivos }(r=0,40, p<0,01) \text { e afetos negativos }(r=-0,22 ; p<0,01)\end{array}$ \\
\hline & $\begin{array}{l}\text { Regressões } \\
\text { múltiplas }\end{array}$ & $\begin{array}{l}\text { O apoio social predisse significativamente satisfação com a vida }(\beta=0,114 ; p<0,05) \text { e afetos } \\
\text { positivos }(\beta=0,346 ; p<0,001) \text {. O autocontrole mediou parcialmente a relação entre o apoio e: } \\
\text { satisfação com a vida }(\beta=0,007 ; p<0,05) \text {, afetos positivos }(\beta=0,099 ; p<0,01) \text {, afetos negativos } \\
(\beta=-0,199 ; p<0,01)\end{array}$ \\
\hline \multirow[b]{2}{*}{$\operatorname{Tian}^{(25)}$} & $\begin{array}{l}\text { Correlações } \\
\text { de Pearson }\end{array}$ & $\begin{array}{l}\text { O bem-estar esteve correlacionado com apoio social provido }(r=0,45 ; p<0,01) \text {, apoio social } \\
\text { recebido }(r=0,40 ; p<0,01) \text {, autoestima ( } \mathrm{r}=0,41 ; \mathrm{p}<0.01) \text { e solidão }(\mathrm{r}=-0,33 ; \mathrm{p}<0,01)\end{array}$ \\
\hline & $\begin{array}{l}\text { Modelagem } \\
\text { de equação } \\
\text { estrutural }\end{array}$ & $\begin{array}{l}\text { O apoio social intergeracional teve efeito direto }(\beta=0,43 ; p<0,05) \text { sobre o BES e indireto }(\beta= \\
0.21, p<0.05 \text { ) através das variáveis autoestima e solidão (Bootstrap estimation) }\end{array}$ \\
\hline $\operatorname{Lou}^{(28)}$ & $\begin{array}{l}\text { Regressões } \\
\text { múltiplas }\end{array}$ & $\begin{array}{l}\text { Na relação com os netos, a satisfação com a vida foi predita pelo apoio emocional recebido ( } \beta= \\
0,14 ; p<0,05) \text { e recíproco }(\beta=-0,13 ; p<0,01) \text { e qualidade de relacionamento }(\beta=0,13 ; p<0,05)\end{array}$ \\
\hline
\end{tabular}




\begin{tabular}{|c|c|c|}
\hline Li et al. ${ }^{(30)}$ & $\begin{array}{l}\text { Regressões } \\
\text { múltiplas }\end{array}$ & $\begin{array}{l}\text { Entre os idosos casados, a variável afetos positivos esteve associada com: apoio em relação ao } \\
\text { cônjuge }(r=0,18 ; p<0,001) \text {, em relação aos filhos }(r=0,30 ; p<0,001) \text { e em relação aos amigos } \\
(r=0,43 ; p<0,001) \text {. A variável afetos negativos esteve associada com: apoio em relação ao } \\
\text { cônjuge }(r=-0,35 ; p<0,001) \text {, em relação aos filhos }(r=-0,27 ; p<0,001) \text { e em relação aos amigos } \\
(r=-0,13 ; p<0,01) \text {. Entre os idosos viúvos ou divorciados, a variável afetos positivos esteve } \\
\text { associada com: apoio em relação aos filhos }(r=0,20 ; p<0,05) \text { e em relação aos amigos }(r= \\
0,41 ; p<0,001) \text {. A variável afetos negativos esteve associada com: apoio em relação aos filhos } \\
(r=-0,34 ; p<0,001) \text { e aos amigos }(r=-0,21 ; p<0,01) \\
\text { Entre idosos casados: O apoio em relação aos amigos foi o único preditor significativo dos } \\
\text { afetos positivos }(\beta=0,32 ; p<0,001) \text { e o apoio em relação com cônjuge foi o único preditor } \\
\text { significativo de afetos negativos }(\beta=-0,26 ; p<0,001) \text {. Entre os viúvos/divorciados: o apoio em } \\
\text { relação aos amigos foi o único preditor significativo dos afetos positivos }(\beta=0,36 ; p<0,001) \text { e } \\
\text { o apoio em relação aos filhos foi o único preditor significativo de afetos negativos }(\beta=-0,29 ; \\
p<0,01)\end{array}$ \\
\hline \multirow[t]{2}{*}{ Peng et al. ${ }^{(31)}$} & $\begin{array}{l}\text { Correlações } \\
\text { de Pearson }\end{array}$ & $\begin{array}{l}\text { A dimensão afetiva do bem-estar subjetivo apresentou correlação direta e significativa com } \\
\text { apoio emocional recebido }(r=0,447 ; p<0,01) \text {, apoio emocional provido }(r=0,377 ; p<0,01) \text { e } \\
\text { apoio instrumental recebido }(r=0,329 ; p<0,01) \text { na relação com os filhos em idosos migrantes; e } \\
\text { apenas com apoio emocional provido aos filhos }(r=0,377 ; p<0,01) \text { em idosos locais }\end{array}$ \\
\hline & $\begin{array}{l}\text { Regressões } \\
\text { múltiplas }\end{array}$ & $\begin{array}{l}\text { A dimensão afetiva do BES pôde ser predita apenas por apoio emocional recebido dos filhos } \\
(\beta=0,37 ; p<0,01) \text { em idosos migrantes; e por apoio emocional provido aos filhos }(\beta=0,26 \text {; } \\
p<0,05) \text { em idosos locais }\end{array}$ \\
\hline \multirow{2}{*}{$\begin{array}{l}\text { Phillips } \\
\text { al. }^{(33)}\end{array}$} & $\begin{array}{l}\text { Correlações } \\
\text { de Pearson }\end{array}$ & $\begin{array}{l}\text { A dimensão afetiva do bem-estar subjetivo apresentou correlação direta e significativa com } \\
\text { satisfação com o apoio recebido de modo geral }(r=0,12 ; p<0,01) \text {, e com satisfação com o } \\
\text { apoio recebido de familiares }(r=0,22 ; p<0,001)\end{array}$ \\
\hline & $\begin{array}{l}\text { Regressões } \\
\text { múltiplas }\end{array}$ & $\begin{array}{l}\text { A dimensão afetiva do BES pôde ser predita pela satisfação com o apoio recebido }(\beta=0,15 ; p< \\
0,001)\end{array}$ \\
\hline $\begin{array}{l}\text { Chalise } \\
\text { al. }^{(34)}\end{array}$ & $\begin{array}{l}\text { Regressões } \\
\text { logísticas }\end{array}$ & $\begin{array}{l}\text { A satisfação com a vida foi predita pelo apoio social recebido do cônjuge }(\beta=0,27 ; p<0,05) \text {, e } \\
\text { apoio provido aos filhos que não coabitam }(\beta=0,44 ; p<0,05) \text { e a parentes }(\beta=-0,26 ; p=0,053) \\
\text { em idosos do sexo masculino. Houve relação significativa entre satisfação com a vida e apoio } \\
\text { provido a filhos que não coabitam }(\beta=0,26 ; \mathrm{p}=0,011) \text { na amostra geral. } \\
\text { Entre os homens a dimensão afetiva do BES foi predita pelo apoio recebido de parentes }(\beta= \\
-0,48 ; p=0,003) \text { e pelo apoio provido aos filhos que coabitam }(\beta=0,31 ; p=0,011) \text {. Na amostra } \\
\text { geral, a dimensão afetiva do BES foi predita pelo apoio recebido de parentes }(\beta=0,18 ; p=0,05)\end{array}$ \\
\hline \multirow[b]{2}{*}{ Chalise $^{(35)}$} & \multirow{2}{*}{$\begin{array}{l}\text { Regressões } \\
\text { múltiplas }\end{array}$} & $\begin{array}{l}\text { Entre os sujeitos da casta Chhetri, a satisfação com a vida foi predita pelos apoios recebido e } \\
\text { provido na relação com o cônjuge }(\beta=0,216 ; p<0,1 \text { e } \beta=0,260 ; p<0,1 \text {, respectivamente). Entre } \\
\text { os sujeitos da casta Newar, a satisfação com a vida foi predita, na relação com os filhos, pelos } \\
\text { apoios recebido ( } \beta \text { filhos que coabitam }=0,343 ; p<0,01 ; \beta \text { filhos que não coabitam }=0,159 ; p< \\
0,05) \text { e provido ( } \beta \text { filhos que coabitam }=0,151 ; p<0,01 ; \beta \text { filhos que não coabitam= }=0,184 ; p< \\
0,01) \text {. O apoio provido aos amigos/vizinhos previu a satisfação com a vida tanto em idosos da } \\
\text { casta Chhetri }(\beta=0,153 ; p<0,05) \text { quanto em idosos da casta Newar }(\beta=0,127 ; p<0,05)\end{array}$ \\
\hline & & $\begin{array}{l}\text { A dimensão afetiva do BES esteve associada, em ambas as castas, com o apoio recebido } \\
\text { do cônjuge }(\beta \text { Chhetri }=0,099 ; p=0,038 ; \beta \text { Newar }=0,132 ; p=0,008) \text { e o apoio provido a ele } \\
\text { ( } \beta \text { Chhetri }=0,117 ; p=0,026 ; \beta \text { Newar }=0,132 ; p=0,001) \text {. O apoio provido a amigos/vizinhos } \\
\text { esteve associado com a dimensão afetiva do BES tanto para sujeitos da casta Chhetri }(\beta=0,141 ; \\
p=0,008) \text { como da casta Newar }(\beta=0,146 ; p=0,006) \text {. Entre os sujeitos da casta Newar, o } \\
\text { apoio recebido dos filhos que coabitam }(\beta=0,127 ; p=0,008) \text {, dos filhos que não coabitam ( } \beta= \\
0,131 ; p=0,008) \text {, de amigos/vizinhos }(\beta=0,191 ; p=0.008) \text { e o apoio provido aos filhos que não } \\
\text { coabitam }(\beta=0,126 ; p=0,008) \text { também estiveram relacionados a um maior nivel da dimensão } \\
\text { afetiva do BES }\end{array}$ \\
\hline
\end{tabular}

Nota: NI: Não informado

\section{Bem-Estar Subjetivo}

No tocante à mensuração do bem-estar subjetivo, cinco estudos avaliaram as três facetas do BES, enquanto dois analisaram exclusivamente o seu componente cognitivo de satisfação com a vida e três, os componentes afetivos. Dentre os instrumentos utilizados para avaliar satisfação com a vida, um estudo ${ }^{(20)}$ aplicou a Escala de Ânimo -(Philadelphia Geriatric Center Morale 
Scale - PGCMS) ${ }^{(21,22)}$, que consiste em 17 itens dicotômicos relacionados à satisfação com a vida. Pontuam-se apenas as respostas afirmativas e atribui-se valor zero para cada resposta "não". A pontuação total varia de 0-17, com uma maior pontuação indicando um maior nível de satisfação com a vida.

Três pesquisas ${ }^{(23-25)}$ utilizaram a Escala de Satisfação com a Vida(26,27) composta por cinco sentenças relacionadas ao construto mensurado, nas quais os participantes indicam o quanto concordam ou discordam de cada uma através de uma escala do tipo Likert, de sete pontos (discordo totalmente a concordo totalmente). Outro autor ${ }^{(28)}$ utilizou uma versão dessa escala, que foi adaptada para o contexto chinês ${ }^{(29)}$.

Para avaliar a dimensão afetiva do BES, a escala mais utilizada foi a Escala de Afetos Positivos e Negativos (Positive Affect and Negative Affect Scale - PANAS ${ }^{(23-25,30)}$. Este instrumento consiste em uma série de palavras que representam diferentes emoções relacionadas ao prazer e ao desprazer, em que o sujeito deve indicar como tem se sentido ultimamente. Os afetos positivos e negativos são corrigidos separadamente e, quanto maior o resultado em cada um, maior o grau daquela característica.

Em um estudo recente ${ }^{(31)}$ optou-se pela Escala Memorial University of Newfoundland Scale of Happiness (MUNSH), desenvolvida para avaliar a experiência afetiva entre os idosos a partir de itens divididos em quatro dimensões: afeição positiva, afeição negativa, experiências de vida positivas e experiências de vida negativas ${ }^{(32)}$. Ainda em relação à avaliação da dimensão afetiva do bem-estar, outra pesquisa ${ }^{(33)}$ utilizou cinco itens da versão chinesa do World Health Organization Quality of Life (WHOQOL).

A versão adaptada do instrumento Life Satisfaction Index K (LSIK) foi aplicada em dois estudos ${ }^{(34,35)}$ que tinham por intuito avaliar tanto o bem-estar cognitivo (satisfação com a vida a longo prazo) quanto o bem-estar afetivo (a curto prazo). A LSIK acessa o BES através da avaliação desses construtos separadamente.

No que concerne à forma de analisar os dados mensurados, dos cinco artigos que avaliaram todos os componentes do BES ( $\mathrm{SV}$, AP e AN), apenas um apresentou medidas separadas para cada uma delas ${ }^{(24)}$. Outros dois obtiveram um escore global de bem-estar a partir da composição entre as três medidas $(\mathrm{BES}=\mathrm{SV}+\mathrm{AP}-\mathrm{AN})^{(23,25)}$. Houve ainda outros dois que apresentaram um escore independente para satisfação com a vida e uma segunda medida composta pelo balanço afetivo (AP - AN) ${ }^{(34,35)}$.

Entre os estudos que avaliaram apenas a experiência afetiva, um estudo(30) apresentou medidas separadas para afetos positivos e afetos negativos, enquanto outros dois estudos ${ }^{(31,33)}$ compuseram uma medida única para a experiência de afetos $(\mathrm{AP}-\mathrm{AN})$.

Duas pesquisas com desenhos metodológicos similares avaliaram a relação entre os componentes do bem-estar subjetivo realizando uma análise fatorial confirmatória que indicou um fator único para as três medidas, o que justificou a postura de adotar uma medida global do BES. Além disso, verificaram a associação entre esse escore único e as três medidas separadamente através da modelagem de equação estrutural. Os resultados do primeiro revelaram que o BES global apresentou associação alta e significativa com a satisfação com a vida e com os afetos positivos $(\beta=0,88, p<0,05$; e $\beta=0,83, p<0,05$, respectivamente) e uma associação fraca com afetos negativos $(\beta=-0,23, p<0,05)^{(23,25)}$. De modo semelhante, verificou-se associações muito fortes e significativas entre o BES Global e a satisfação com a vida $(\beta=0,83, p<0,05)$ e também entre BES Global e os afetos $\operatorname{positivos}(\beta=0,91, p<0,05)$, sendo novamente encontrada associação fraca com afetos negativos $(\beta=-0,15, p<0,05)$.

Pesquisa anterior ${ }^{(24)}$ observou fraca correlação entre satisfação com a vida e afetos positivos $(r=0,182, p<0,01)$ e moderada entre satisfação com a vida e afetos negativos $(r=-0,34, p<0,01)$. A correlação entre os afetos positivos e negativos não foi significativa. As relações entre essas variáveis, entretanto, não foram estudadas nas regressões múltiplas realizadas.

\section{Apoio Social}

Em relação aos instrumentos utilizados para avaliar o apoio social, aqueles construídos pelos autores com base nos fundamentos conceituais do construto foram os mais predominantes $(60 \%)$. Um estudo ${ }^{(34)}$ elaborou duas escalas independentes para avaliar a frequência de apoio recebido e provido, bem como para investigar o vínculo do idoso com as pessoas envolvidas na relação de ajuda, e um estudo posterior utilizou essa escala já construída ${ }^{(35)}$.

Outro estudo ${ }^{(30)}$ avaliou o apoio social através de três questões que avaliavam a frequência de contato com familiares e amigos, o nível de apoio recebido deles e o nível de apoio provido a eles. As questões eram fechadas e as opções de resposta variavam entre 1 (muito raramente) e 5 (muito frequentemente). O escore final era obtido através da média dos três itens.

Outra pesquisa avaliou a mensuração do grau de satisfação do idoso em relação ao apoio recebido de cada fonte separadamente, a saber: cônjuge, filhos e amigos; sendo utilizada uma escala do tipo item único, em que o participante escolhia sua resposta dentro de um intervalo que variava entre 1 (muito insatisfeito) e 5 (muito satisfeito) ${ }^{(33)}$.

Um estudo ${ }^{(28)}$ avaliou o apoio em relação aos netos a partir de três domínios independentes: apoio emocional recebido, apoio recíproco e qualidade de relacionamento. O primeiro foi mensurado pela pergunta "Quão frequente você sente que seu neto provê apoio emocional para você?". A resposta era apontada em uma escala do tipo Likert, de cinco pontos (nunca a sempre). O apoio recíproco foi avaliado por dois itens "Quando você tem uma decisão importante a tomar, você discute isso com seus netos?" e "Quando seus netos têm uma decisão importante a tomar, eles pedem sua ajuda?". Ambas permitiam um formato de resposta Sim / Não. Aqueles que relataram 'sim' para ambas as perguntas foram agrupados em um grupo de apoio recíproco. O restante foi agrupado num grupo de apoio não recíproco. Por fim, a qualidade do relacionamento com os netos era 
verificada através de uma escala de sete itens que dizia respeito às consequências positivas trazidas pelo relacionamento, como novas percepções, continuidade familiar e autoestima.

Um mesmo grupo de pesquisadores elaborou duas escalas, de 28 itens cada, para avaliar a frequência dos apoios recebido e provido no relacionamento do idoso com seus filhos. Adaptaram ainda o instrumento Perceived Social Support Scale (PSSS) para acessar o apoio percebido em relação às pessoas de fora do contexto familiar ${ }^{(31)}$.

Entre os artigos que utilizaram escalas desenvolvidas e validadas por outros autores, destacam-se: a Medical Outcomes Study Social Support Survey scales ${ }^{(20)}$, Perceived Social Support Scale ${ }^{(23)}$, Chinese Social Support Rating Scale ${ }^{(24)}$ e Intergenerational Social Support Scale ${ }^{(25)}$.

Em síntese, cinco artigos mensuraram tanto o apoio social provido como o apoio social recebido. Destes, três analisaram os tipos de apoio separadamente ${ }^{(31,34,35)}$ e dois obtiveram um escore único a partir da combinação dos apoios ${ }^{(25,30)}$. Dois estudos

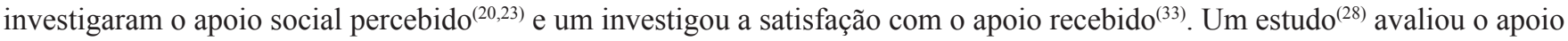
emocional recebido e a qualidade do relacionamento com os netos. Um outro estudo analisou o apoio informacional recebido e provido, dividindo os idosos em dois grupos de acordo com suas respostas (apoio recíproco e apoio não-recíproco) enquanto outra pesquisa $^{(24)}$ declarou avaliar os apoios subjetivos, objetivos e a forma de utilização do apoio, embora não detalhassem o que significava cada uma dessas dimensões. O escore do apoio social geral era obtido através da combinação das medidas.

No que tange aos sujeitos envolvidos na relação de ajuda, nove estudos investigaram o apoio informal, sendo caracterizado como uma avaliação do apoio social percebido de modo geral sem especificar a fonte do apoio em questão, ou seja, diante de cada item do instrumento, o idoso indicava o quanto acreditava que determinado tipo de apoio estaria disponível caso precisasse, sem apontar o responsável por esse provimento ${ }^{(20)}$.

\section{Apoio Social e Bem-Estar Subjetivo}

Apenas um artigo não realizou análises estatísticas para verificar a consistência interna dos instrumentos na amostra estudada. Os demais apresentaram bons indicadores de confiabilidade. Como se pode observar no Quadro II, todos os instrumentos obtiveram valores de Alpha acima de 0,7, considerado o mínimo aceitável pela literatura ${ }^{(36)}$. Esses resultados indicam que os instrumentos utilizados para avaliar o apoio social e o BES têm boa consistência interna, sendo confiáveis para medir as variáveis propostas.

Quanto aos resultados, todos os estudos selecionados encontraram associações entre apoio social e bem-estar. Um estudo ${ }^{(33)}$ encontrou que idosos que relataram estarem mais satisfeitos com o apoio recebido de modo geral indicavam uma experiência afetiva mais positiva em comparação com aqueles que relataram baixo nível de satisfação com o apoio.

Nos demais estudos, as associações entre AS e BES tenderam a variar, tanto em termos de magnitude como em termos de significância estatística, quando se levava em consideração a cultura local e o tipo de relacionamento dos envolvidos nas trocas sociais. Um estudo ${ }^{(35)}$, por exemplo, avaliou o impacto do AS sobre o BES considerando não apenas o nível de apoio autorrelatado, mas também os tipos de vinculo presentes nas relações de ajuda (cônjuge, filhos e amigos/ vizinhos). Considerou ainda o aspecto cultural ao avaliar e comparar duas amostras de castas diferentes na cidade de Katmandu, Nepal (Chhetri e Newar).

Neste caso, os idosos da casta Chhetri que relataram receber e prover apoio na relação com o cônjuge apresentaram maior satisfação com a vida. Já entre os sujeitos da casta Newar, a satisfação com a vida foi maior para aqueles que recebiam e proviam apoio na relação com os filhos (morassem eles juntos ou não). Prover apoio aos amigos e vizinho aumentou a satisfação com a vida tanto em idosos da casta Chhetri como em idosos da casta Newar ${ }^{(35)}$.

Quanto à relação entre o apoio e a dimensão afetiva do BES, idosos que relataram receber e prover apoio na relação com o cônjuge apresentaram uma experiência afetiva mais positiva em ambas as castas. Da mesma forma, aqueles que relataram prover apoio a amigos e vizinhos também demonstraram um alto nível de experiência afetiva positiva, fossem eles da casta Chhetri ou Newar. Contudo, o apoio recebido dos filhos que coabitam e dos filhos que não coabitam, de amigos/vizinhos e o apoio provido aos filhos que não coabitam melhoraram a experiência afetiva apenas em indivíduos da casta Newar ${ }^{(35)}$.

Um grupo de autores ${ }^{(31)}$, ao estudarem uma amostra composta por idosos migrantes que residiam Shenzhen (China) e idosos locais, encontraram que idosos migrantes que recebiam apoio emocional dos filhos tendiam a experimentar mais afetos positivos e, por conseguinte, menos afetos negativos. Entre os idosos locais, o que proporcionava uma maior qualidade na experiência afetiva era prover apoio emocional aos filhos.

Os resultados encontrados em uma amostra de idosos residentes em Beijing (China) ${ }^{(30)}$ revelaram que quanto mais se recebia apoio dos amigos e quanto mais se provia apoio a eles, mais afetos positivos eram vivenciados pelos idosos, fossem eles casados, viúvos ou divorciados. Além disso, receber e prover apoio na relação com o cônjuge protegeu o idoso casado contra os afetos negativos. Entre os idosos viúvos e divorciados, o apoio recebido e provido em relação aos filhos substituiu o apoio do cônjuge quanto ao papel protetor frente aos afetos negativos.

Dois estudos, embora apontassem para relações positivas entre o apoio social e o bem-estar, encontraram também associações negativas entre essas variáveis, sendo constatado que idosos nepaleses do sexo masculino que recebiam apoio do cônjuge e proviam apoio aos filhos que coabitam, tendiam a ter uma satisfação com a vida mais elevada. Em contrapartida, a 
satisfação com a vida destes idosos tendia a diminuir com o provimento de apoio a parentes. Além disso, estes também tinham a dimensão afetiva comprometida quando recebiam apoio de parentes $^{(34)}$.

Em uma amostra de idosos chineses (Hong Kong), aqueles que recebiam apoio emocional de seus netos apresentavam maior satisfação com a vida. Entretanto, os participantes que indicaram a existência de apoio informacional recíproco na relação com os netos (davam e recebiam conselhos) tinham menor satisfação com a vida ${ }^{(28)}$.

Duas pesquisas apontaram ainda o efeito mediador do apoio social sobre a relação do BES com uma terceira variável. Os resultados encontrados nesses estudos ${ }^{(20)}$ revelaram que o tipo de arranjo domiciliar exerceu influência sobre a satisfação com a vida do idoso através do apoio social percebido. Deste modo, o arranjo domiciliar no qual o idoso está inserido impactou de forma direta sua percepção de apoio e esta, por sua vez, impactou na sua satisfação com a vida, enquanto outra pesquisa ${ }^{(23)}$ evidenciou que o apoio social percebido funcionou como uma variável mediadora entre a rede social e o bem-estar subjetivo. Portanto, não bastava apenas que o idoso tivesse contato com muitas pessoas em sua vida, elas precisavam representar prováveis fontes de apoio para poder impactar positivamente o bem-estar dos idosos.

Outros dois estudos encontraram um impacto positivo do apoio social sobre o bem-estar subjetivo através de outras variáveis. Os idosos chineses que recebiam e proviam apoio na relação com seus filhos relataram maior autoestima e menor solidão, consequentemente, apresentavam um maior nível de $\mathrm{BES}^{(25)}$. Neste estudo, a autoestima e a solidão funcionaram como pontes entre o apoio social e o bem-estar. Em outro estudo, o autocontrole mediou parcialmente a relação entre o apoio e satisfação com a vida, os afetos positivos, e os afetos negativos na amostra estudada ${ }^{(24)}$.

Em suma, quatro tipos de impactos do AS sobre o BES foram observados: (a) direto e positivo, quando o apoio aumenta os níveis de satisfação com a vida e de afetos positivos, e diminui o nível de afetos negativos; (b) direto e negativo, quando o apoio diminui os níveis de satisfação com a vida e de afetos positivos, e aumenta o nível de afetos negativos; (c) direto e mediador, quando o apoio social se coloca entre o bem-estar e uma terceira variável, mediando a relação entre elas; e (d) indireto e mediado, quando o apoio social exerce influência sobre o bem-estar através de uma terceira variável.

\section{DISCUSSÃO}

$\mathrm{Na}$ presente revisão observou-se que todos os estudos foram realizados em países orientais. Uma possível explicação para esse fato pode ter base cultural, tendo em vista que as sociedades pertencentes a esse hemisfério são predominantemente coletivistas, enfatizando os recursos sociais, os aspectos da interação interpessoal e as relações intra e inter-grupais ${ }^{(37-39)}$. Somado a isso, há ainda o envelhecimento populacional. Apesar da população de idosos mostrar crescimento em todo o mundo, o envelhecimento populacional na Ásia acontece em ritmo ainda mais acelerado ${ }^{(40)}$. Estima-se que a parcela da população com mais de 65 anos na Ásia será de $16,8 \%$ até o ano de $2050^{(41)}$.

Os instrumentos mais utilizados para avaliação dos componentes do bem-estar subjetivo foram o Satisfaction With Life Scale - SWLS ${ }^{(26)}$, para mensurar a dimensão cognitiva do BES, e o Positive Affect and Negative Affect Scales - PANAS ${ }^{(42)}$, para avaliar a dimensão afetiva. Embora elaboradas na década de 1980, essas escalas continuam sendo utilizadas em diversos estudos sobre o bem-estar e têm demonstrando bons indicadores de validade e confiabilidade, podendo ser consideradas padrões ouro nesse campo ${ }^{(43)}$.

O mesmo tipo de resultado não aconteceu com os instrumentos utilizados para acessar a variável apoio social e suas dimensões. Diferentes escalas foram utilizadas, ressaltando-se a prevalência daquelas construídas pelos autores com base nos fundamentos conceituais do construto. Identificaram-se seis estudos, entre 10 avaliados, que utilizaram escalas próprias, enquanto outros estudos utilizaram escalas já existentes após realizarem adaptações nas mesmas. Uma possível justificativa para esse ocorrido é a sensibilidade do apoio social às diferenças culturais presentes nos diversos grupos estudados, demandando a construção/ adaptação de instrumentos que fossem ecologicamente válidos ${ }^{(44,45)}$.

Em relação à associação entre apoio social e bem-estar subjetivo, a literatura revisada demonstrou, majoritariamente, um impacto positivo do primeiro sobre o segundo, fosse ele direto ou indireto, indicando que quanto maior o nível de apoio, maior o nível de bem-estar subjetivo experimentado pelos idosos. Esses resultados foram concernentes ao modelo de efeito direto (ou principal), o qual considera que o apoio exerce efeitos diretos e indiretos sobre o bem-estar dos indivíduos ${ }^{(46)}$. Além disso, os resultados foram consistentes com resultados anteriores ${ }^{(6,47-49)}$. Não foram encontradas evidencias que corroborassem o papel protetor do apoio frente às circunstancias adversas da vida (modelo do efeito amortecedor ou buffer) ${ }^{(46)}$.

Vale ressaltar a influência positiva do apoio social provido pelo idoso sobre seu bem-estar subjetivo nos artigos analisados. Em geral, presume-se que os idosos precisam de apoio para satisfazer suas necessidades, isto é, o idoso é visto de forma passiva, como aquele que demanda por cuidados. A literatura, no entanto, aponta que os idosos não apenas podem ser fontes de apoio, como também experimentam benefícios ao ajudarem outras pessoas. Esses resultados caminham no sentido de promover uma mudança de perspectiva ao olhar para os mais velhos, vendo-os como sujeitos que podem ser ativos, dando e recebendo recursos para a promoção do seu bem-estar ${ }^{(6,47)}$.

As associações negativas entre apoio provido aos parentes e satisfação com a vida e, ainda entre apoio recebido de parentes e experiência afetiva ${ }^{(34)}$, podem ser explicadas culturalmente, entendendo que homens idosos da região estudada têm percepções 
negativas sobre dar e receber apoio de parentes. Contudo, as razões por trás dessas descobertas precisam de mais investigações no futuro.

Os resultados encontrados em uma pesquisa ${ }^{(28)}$ também apontaram para um lado negativo do apoio social. No estudo em questão, os idosos que relataram apoio informacional recíproco em relação aos seus netos (davam conselho a eles e os procuravam para pedir conselhos) demonstraram um menor nível de satisfação com a vida. Na perspectiva do autor, buscar aconselhamentos com os netos viola a expectativa do papel social do idoso, o qual, devido às vivências, deveria desempenhar uma figura de sabedoria e autoridade.

O autor entende que não é esperado que avós busquem conselhos de seus netos diante dos desafios da vida. Novamente, a cultura parece desempenhar uma função importante na forma como o apoio social é interpretado pelos sujeitos da relação. Entretanto, em relação ao apoio informacional, o autor divide os idosos entre dois grupos: "apoio recíproco" e "apoio não recíproco", não diferenciando, neste último, os idosos que relataram somente buscar conselhos de seus netos daqueles que relataram somente serem procurados para aconselhamento ${ }^{(28)}$. Deste modo, mais pesquisas precisam ser realizadas no sentido de uma maior compreensão dessa questão.

Assim, dois artigos mostraram um efeito negativo do apoio social sobre o bem-estar. Contudo, essa influência foi delimitada a determinados tipos de apoio em relacionamentos interpessoais específicos (entre os idosos e seus netos, ou entre os idosos e seus parentes).

De modo geral, todos os estudos encontraram relação positiva entre apoio e bem-estar subjetivo, mesmo aqueles que encontraram também o efeito negativo. Logo, a tendência dos resultados dos artigos avaliados foi a de que o apoio eleva o BES dos idosos, levando-os a avaliar suas vidas de modo mais positivo e a experimentar mais afetos agradáveis e menos afetos desprazerosos. As diferenças significativas entre as subescalas do bem-estar subjetivo e as diferentes facetas do apoio social corroboram a importância da distinção dos componentes cognitivos e afetivos no acesso e na interpretação desse construto também na velhice ${ }^{(50,51)}$.

Quanto aos resultados do presente estudo, também foram encontradas limitações. Em alguns casos, os elementos teóricos e a descrição dos instrumentos não foram suficientemente explorados pelos autores, o que impediu uma análise mais acurada desses aspectos.

Além disso, as medidas de apoio social utilizadas foram subjetivas, representando o reconhecimento da disponibilidade de apoio pelo próprio sujeito. Portanto, baixos níveis de apoio social autorrelatados não indicam necessariamente que o idoso esteja recebendo menos apoio. Variáveis intrínsecas ao individuo podem levá-lo a não se sentir apoiado mesmo que receba apoio ${ }^{(52)}$. O cenário no qual o apoio é provido, mas o receptor não o reconhece, é denominado pela literatura como apoio invisível ${ }^{(53)}$.

Espera-se que este estudo contribua para a implementação de avanços teóricos e metodológicos que auxiliem nessa questão, além de fomentar a implementação de intervenções no sentido de promover o bem-estar e a qualidade de vida entre os idosos

\section{CONCLUSÃO}

Os resultados dessa revisão apontam que a relação entre apoio social e bem-estar subjetivo é, de modo geral, positiva. A tendência dos resultados dos artigos avaliados foi a de que o apoio eleva o BES dos idosos, levando-os a avaliar suas vidas de modo mais positivo e a experimentar mais afetos agradáveis. Contudo, esses resultados podem ter sido afetados por aspectos culturais, tendo em vista que apenas idosos de países orientais foram incluídos nas amostras, sinalizando a necessidade de estudos em outras populações.

Ademais, foi evidenciada a necessidade de desenvolvimento de pesquisas que investiguem a influencia do apoio social sobre o bem-estar subjetivo a partir de delineamentos experimentais, pois permitirá um melhor entendimento da relação entre esses dois construtos.

\section{REFERÊNCIAS}

1. Coleta JAD, Lopes JEF, Coleta MFD. Felicidade, bem-estar subjetivo e variáveis sociodemográficas, em grupos de estudantes universitários. Psico USF. 2012;17(1):129-39.

2. Diener E. The remarkable changes in the science of subjective well-being. Perspect Psychol Sci. 2013;8(6):663-6.

3. Layous K, Zanon C. Avaliação da felicidade subjetiva: para além dos dados de autorrelato. In: Hutz CS. Avaliação em psicologia positiva. Porto Alegre: Artmed; 2014. p. 23-42.

4. Albuquerque AS, Tróccoli BT. Desenvolvimento de uma escala de bem-estar subjetivo. Psicol Teor Pesqui. 2004;20(2):15364.

5. Teixeira INDO, Neri AL. Envelhecimento bem-sucedido: uma meta no curso da vida. Psicologia USP. 2008;19(1):81-94. 
6. Siedlecki KL, Salthouse TA, Oishi S, Jeswani S. The relationship between social support and subjective well-being across age. Soc Indic Res. 2014;117(2):561-76.

7. Cho J, Martin P, Poon LW. Successful aging and subjective well-being among oldest-old adults. Gerontologist. 2015;55(1):132-43.

8. Oliveira SF, Queiroz MIN, Costa MLA. Bem estar subjetivo na terceira idade. Motricidade. 2012;8(Supl 2):1038-47.

9. Cardoso MCS, Ferreira MC. Envolvimento religioso e bem-estar subjetivo em idosos. Psicol Ciênc Prof. 2009;29(2):38093.

10. Woyciekoski C, Stenert F, Hutz CS. Determinantes do bem-estar subjetivo. Psico. 2012;43(3):280-8.

11. Olson EA, Fanning JT, Awick EA, Chung HD, McAuley E. Differential trajectories of well $\square$ being in older adult women: the role of optimism. Appl Psychol Health Well Being. 2014;6(3):362-80.

12. Carmel S, Raveis VH, O'Rourke N, Tovel H. Health, coping and subjective well-being: results of a longitudinal study of elderly Israelis. Aging Ment Health. 2017;21(6):616-23.

13. Simone PM, Haas AL. Frailty, leisure activity and functional status in older adults: Relationship with subjective well being. Clinical Gerontologist. 2013;36(4):275-93.

14. Carr D, Freedman VA, Cornman JC, Schwarz N. Happy marriage, happy life? Marital quality and subjective well being in later life. J Marriage Fam. 2014;76(5):930-48.

15. Golçalves TR, Pawlowski J, Bandeira DR, Piccinini CA. Avaliação de apoio social em estudos brasileiros: aspectos conceituais e instrumentos. Ciênc Saúde Coletiva. 2011;16(3):1755-69.

16. Griep RH, Chor D, Faerstein E, Werneck GL, Lopes CS. Validade de constructo de escala de apoio social do Medical Outcomes Study adaptada para o português no Estudo Pró-Saúde. Cad Saúde Pública. 2005;21(3):703-14.

17. Mas-Expósito L, Amador-Campos JA, Gómez-Benito J, Lalucat-Jo L. Considering variables for the assignment of patients with schizophrenia to a case management programme. Community Ment Health J. 2013;49(6):831-40.

18. Shiba K, Kondo N, Kondo K. Informal and formal social support and caregiver burden: the AGES Caregiver Survey. J Epidemiol. 2016;26(12):622-8.

19. Law M, Stewart D, Pollock N, Letts L, Bosch J, Westmorland M. Guidelines for critical review form: quantitative studies [acesso em 2017 Jan 09]. Disponível em: https://srs-mcmaster.ca/wp-content/uploads/2015/05/Guidelines-for-CriticalReview-Form-Quantitative-Studies.pdf

20. Kooshiar H, Yahaya N, Hamid TA, Samah AA, Jou VS. Living arrangement and life satisfaction in older Malaysians: the mediating role of social support function. PloS one. 2012;7(8):e43125

21. Lawton MP. The Philadelphia Geriatric Center Morale Scale: a revision. J Gerontol. 1975;30(1):85-9.

22. Ma L, Green KE, Cox EO. Stability of the Philadelphia Geriatric Center Morale Scale: a multidimensional item response model and rasch analysis. J Applied Gerontol. 2010;29(4):475-93.

23. Wang X. Subjective well-being associated with size of social network and social support of elderly. J Health Psychol. 2014;21(6):1037-42.

24. Tu Y, Yang Z. Self-control as mediator and moderator of the relationship between social support and subjective well-being among the Chinese elderly. Social Indicators Res. 2016;126(2):813-28.

25. Tian Q. Intergeneration social support affects the subjective well-being of the elderly: Mediator roles of self-esteem and loneliness. J Health Psychol. 2016;21(6):1137-44.

26. Diener E, Emmons RA, Larsen RJ, Griffin S. The satisfaction with life scale. J Pers Assess. 1985;49(1):71-5.

27. Pavot W, Diener E. Review of the satisfaction with life scale. Psychol Assess. 1993;5(2):164-72.

28. Lou VW. Life satisfaction of older adults in Hong Kong: The role of social support from grandchildren. Social Indicators Res. 2010;95(3):377-91.

29. Lou VWQ, Chi I, Mjelde-Mossey LA. Development and validation of a life satisfaction scale for Chinese elders. Int J Aging Hum Dev. 2008;67(2):149-70. 
30. Li H, Ji Y, Chen T. The roles of different sources of social support on emotional well-being among Chinese elderly. PloS One. 2014;9(3):e90051.

31. Peng H, Mao X, Lai D. East or West, home is the best: Effect of intergenerational and social support on the subjective wellbeing of older adults: a comparison between migrants and local residents in Shenzhen, China. Ageing Int. 2015;40(4):37692.

32. Kosma A, Stones MJ. The measurement of happiness: development of the Memorial University of Newfoundland Scale of Happiness (MUNSH). J Gerontol. 1980;35(6):906-12.

33. Phillips DR, Siu OL, Yeh AG, Cheng KH. Informal social support and older persons' psychological well-being in Hong Kong. J Cross Cult Gerontol. 2008;23(1):39-55.

34. Chalise HN, Saito T, Takahashi M, Kai I. Relationship specialization amongst sources and receivers of social support and its correlations with loneliness and subjective well-being: A cross sectional study of Nepalese older adults. Arch Gerontol Geriatr. 2007;44(3):299-314.

35. Chalise HN. Social Support and its Correlation with Loneliness and Subjective Well-being: A Cross-cultural Study of Older Nepalese Adults. Asian Soc Work Policy Rev. 2010;4(1):1-25.

36. Hora HRM, Monteiro GTR, Arica J. Confiabilidade em questionários para qualidade: um estudo com o Coeficiente Alfa de Cronbach. Produto \& Produção. 2010;11(2):85-103.

37. Ferreira MC, Assmar EML, Souto SO. O individualismo e o coletivismo como indicadores de culturas nacionais: convergências e divergências teórico-metodológicas. Psicol Estud. 2002;7(1):81-9.

38. Gouveia VV, Andrade JD, Milfont TL, Queiroga F, Santos WD. Dimensões normativas do individualismo e coletivismo: É suficiente a dicotomia pessoal vs. social. Psicol Reflex Crít. 2003;16(2):223-34.

39. Keshtiari N, Kuhlmann M. The Effects of culture and gender on the recognition of emotional speech: evidence from persian speakers living in a collectivist society. Int J Society Culture Language. 2016;4(2):71-86.

40. Donehower G, Fürnkranz-Prskawetz A, Lee RD, Lee SH, Mason A, Miller T, et al. Population change and the economic security of older people in Asia. National Transfer Accounts Bull. 2016;10:1-4.

41. Gupta A, Mohan U, Singh SK, Manar MK, Tiwari SC, Singh VK. Screening depression among elderly in a city of Southeast Asia. J Clin Diagn Res. 2015;9(9):LC01-5.

42. Watson D, Clark LA, Tellegen A. Development and validation of brief measures of positive and negative affect: the PANAS scales. J Pers Soc Psychol. 1988;54(6):1063-70.

43. Jovanović V. A bifactor model of subjective well-being: A re-examination of the structure of subjective well-being. Pers Individ Dif. 2015;87:45-9.

44. Winemiller DR, Mitchell ME, Sutliff J, Cline DI. Measurement strategies in social support: a descriptive review of the literature. J Clin Psychol. 1993;49(5):638-48.

45. Antunes C, Fontaine AM. Percepção de apoio social na adolescência: análise fatorial confirmatória da escala Social Support Appraisals. Paidéia. 2005;15(32):355-66.

46. Cohen S, Wills TAS. Stress, Social Support, and the Buffering Hypothesis. Psychol Bulletin. 1985;98(2):310-57.

47. Guedea MTD, Albuquerque FJB, Tróccoli BT, Noriega JAV, Seabra MAB, Guedea RLD. Relação do bem-estar subjetivo, estratégias de enfrentamento e apoio social em idosos. Psicol Reflex Crít. 2006;19(2):301-8.

48. Siewert K, Antoniw K, Kubiak T, Weber H. The more the better? The relationship between mismatches in social support and subjective well-being in daily life. J Health Psychol. 2011;16(4)621-31.

49. Zhao J, Kong F, Wang Y. Shyness and subjective well-being: The role of emotional intelligence and social support. Social Indicators Research. 2013;114(3):891-900.

50. Busseri MA, Sadava SW. A review of the tripartite structure of subjective well-being: Implications for conceptualization, operationalization, analysis, and synthesis. Pers Soc Psychol Rev. 2011;15(3):290-314.

51. Busseri MA. Toward a resolution of the tripartite structure of subjective well being. J Pers. 2015;83(4):413-28.

52. Sarason IG. Social support: theory, research and applications. Washington: NATO ASI Series; 2013. 
53. Ditzen B, Heinrichs M. Psychobiology of social support: the social dimension of stress buffering. Restor Neurol Neurosci. 2014;32(1):149-62.

\section{Endereço para correspondência:}

Luiz Fellipe Dias da Rocha

Universidade do Estado do Rio de Janeiro - UERJ

Centro de Tecnologia e Ciências

Rua São Francisco Xavier, 524/ Sala 2145 F

Bairro: Maracanã

CEP: 20550-900 - Rio de Janeiro - RJ - Brasil

E-mail: felliperocha.uerj@gmail.com 